\title{
Effects of combination therapy using hypothermia and erythropoietin in a rat model of neonatal hypoxia-ischemia
}

\author{
Annie Y. Fang ${ }^{1,2}$, Fernando F. Gonzalez ${ }^{2,3}$, R. Ann Sheldon ${ }^{1,2}$ and Donna M. Ferriero ${ }^{1-3}$
}

BACKGROUND: Hypoxic-ischemic (HI) injury to the developing brain remains a major cause of morbidity. Hypothermia is effective but does not provide complete neuroprotection, prompting a search for adjunctive therapies. Erythropoietin (Epo) has been shown to be beneficial in several models of neonatal $\mathrm{HI}$. This study examines combination hypothermia and treatment with erythropoietin in neonatal rat $\mathrm{HI}$.

METHODS: Rats at postnatal day 7 were subjected to $\mathrm{HI}$ (Vannucci model) and randomized into four groups: no treatment, hypothermia alone, Epo alone, or hypothermia and Epo. Epo $(1,000 \mathrm{U} / \mathrm{kg})$ was administered in three doses: immediately following $\mathrm{HI}$, and $24 \mathrm{~h}$ and $1 \mathrm{wk}$ later. Hypothermia consisted of whole-body cooling for $8 \mathrm{~h}$. At 2 and 6 wk following $\mathrm{HI}$, sensorimotor function was assessed via cylinder-rearing test and brain damage by injury scoring. Sham-treated animals not subjected to HI were also studied.

RESULTS: Differences between experimental groups, except for Epo treatment on histopathological outcome in males, were not statistically significant, and combined therapy had no adverse effects.

CONCLUSION: No significant benefit was observed from treatment with either hypothermia or combination therapy. Future studies may require older animals, a wider range of functional assays, and postinsult assessment of injury severity to identify only moderately damaged animals for targeted therapy.

$\mathrm{H}$ ypoxic-ischemic (HI) injury to the developing brain contributes significantly to mortality and long-term morbidity. Neonatal encephalopathy occurs in 3-5 in 1,000 live births, with moderate or severe HI encephalopathy (HIE) occurring in $0.5-1$ per 1,000 live births. Despite advances in newborn intensive care, $\sim 10-60 \%$ of HIE-affected infants die, and at least $25 \%$ of those who survive exhibit lifelong neurological impairment, with common outcomes of epilepsy, cerebral palsy, and mental retardation (1). Although there are no established interventions that fully treat neonatal HIE, many potential therapies that may prevent injury progression and enhance repair are under investigation (2).

Therapeutic hypothermia has emerged as the first treatment to provide neuroprotection in neonatal HIE in multiple randomized controlled trials (3-5). A variety of mechanisms have been implicated in the effects of hypothermia, including cell death inhibition, cerebral metabolic rate reduction, and diminished release of excitotoxins and reactive oxygen species (6). As reviewed in a recent meta-analysis, hypothermia was shown to significantly reduce mortality and neurodevelopmental disability at $18 \mathrm{mo}$ in human neonates cooled to $33-35^{\circ} \mathrm{C}$ for $72 \mathrm{~h}$ within the first $6 \mathrm{~h}$ of life (5). The rationale for human trials emerged from animal studies of perinatal asphyxia, notably in lamb and piglet models, in which early initiation of hypothermia improved histological and functional outcomes $(7,8)$. Brief hypothermia provided partial neuroprotection, whereas prolonged hypothermia (for 24-72 h) improved longterm behavioral performance (9).

Although hypothermia has been adopted as standard of care in many clinical units for infants with HIE (10), it does not provide complete neuroprotection, prompting urgent investigation into additional agents that may further improve outcomes. One such agent is erythropoietin (Epo), which has recently been shown to preserve brain structure and function in several studies of rodent neonatal stroke (11-13) and some studies of rodent HI (14-16). Although Epo was originally identified for its role in erythropoiesis, it has since been shown to have a wide range of functions that may account for its neuroprotective effects, including cell death inhibition, immune response modulation, initiation of angiogenesis, and promotion of neurogenesis, thereby enhancing repair (17). In human neonates with HIE, large-scale trials have yet to be performed, but some early clinical studies have demonstrated the safety and benefit of Epo treatment at 18 mo $(18,19)$.

Although both hypothermia and Epo show great potential as treatments for neonatal HIE, there is much controversy in the literature regarding optimization of these treatments in the neonatal rodent $\mathrm{HI}$ model. For hypothermia, a wide variety of target temperatures, ranging from 22 to $35^{\circ} \mathrm{C}$, and durations, from 3 to $72 \mathrm{~h}$, have been evaluated with mixed results (20-22). Similarly, optimal dosing and timing of Epo treatment in the neonatal rodent $\mathrm{HI}$ model remains under investigation, with most studies showing maximum benefit with doses between 1,000 and $5,000 \mathrm{U} / \mathrm{kg}$ administered in a multidose regimen 
between three and five times $(15,16,23)$. In addition, sexspecific differences in treatment response to both hypothermia and Epo have been observed $(24,25)$. Using a rodent model of neonatal $\mathrm{HI}$, we evaluated the in vivo effects of whole-body cooling to $32^{\circ} \mathrm{C}$ and multidose Epo administered at 1,000 U/kg and assessed whether combination therapy with both agents would improve functional and structural outcomes beyond either treatment alone.

\section{RESULTS}

\section{Survival Rates and Weights}

A total of 96 animals underwent the HI procedure: 11 animals died posthypoxia and 85 survived. One animal that had been allocated to the hypothermia-only treatment group died during the 2 -wk monitoring period. A total of 19 sham-treated animals underwent anesthesia, with no mortalities. At $1 \mathrm{wk}$ following HI, there was a significant difference between the weights of experimental animals and sham-treated animals $(P=0.0181)$, but no differences between treatment groups (Table 1$)$. The differences in the final weights of animals at killing were not statistically significant.

\section{Maintenance of Cranial Temperature}

Mean cranial skin temperature was significantly different between hypothermic and normothermic animals $(P<0.0001)$ after 4 and $8 \mathrm{~h}$ of temperature modulation. Mean cranial skin temperature in the hypothermic animals was $30.80 \pm 0.25^{\circ} \mathrm{C}$ $(n=40)$, whereas mean temperature in normothermic animals was $33.79 \pm 0.39^{\circ} \mathrm{C}(n=29)$ after $4 \mathrm{~h}$ of temperature modulation (Table 2). Temperature measurements taken at $4 \mathrm{~h}$ were not significantly different from those taken at $8 \mathrm{~h}$.

\section{Blood Glucose Measurements}

Blood glucose samples following temperature modulation were significantly different between hypothermic and normothermic

Table 1. Animal weights at $P 8$ and before killing

\begin{tabular}{llcl}
\hline Weight & Hypothermic & Normothermic & Sham-treated \\
\hline $24 \mathrm{~h} \mathrm{Post}-\mathrm{HI}^{*}$ & $26.72 \pm 3.12 \mathrm{~g}$ & $26.82 \pm 3.52 \mathrm{~g}$ & $29.24 \pm 3.443 \mathrm{~g}$ \\
2 wk Post-HI & $43.93 \pm 6.62 \mathrm{~g}$ & $45.57 \pm 2.64 \mathrm{~g}$ & $46.25 \pm 4.61 \mathrm{~g}$ \\
6 wk Post-HI & $193.8 \pm 50.1 \mathrm{~g}$ & $192.6 \pm 40.7 \mathrm{~g}$ & $217.9 \pm 33.7 \mathrm{~g}$ \\
\hline
\end{tabular}

$\mathrm{HI}$, hypoxia-ischemia; $\mathrm{P}$, postnatal day. ${ }^{*} P<0.05$.

Table 2. Temperature and blood glucose during temperature modulation

\begin{tabular}{lcc}
\hline & Hypothermic & Normothermic \\
\hline Cranial temperature & & \\
After 4h of temperature & $30.80 \pm 0.28^{\circ} \mathrm{C}(n=40)$ & $33.79 \pm 0.39^{\circ} \mathrm{C}(n=29)$ \\
modulation & & \\
After 8h of temperature & $30.74 \pm 0.31^{\circ} \mathrm{C}(n=35)$ & $33.95 \pm 0.33^{\circ} \mathrm{C}(n=21)$ \\
modulation & \\
Blood glucose \\
$\begin{array}{l}\text { After 8h of temperature } \\
\text { modulation }\end{array}$ & $126.2 \pm 5.191(n=35)$ & $108.9 \pm 4.044(n=25)$ \\
\hline
\end{tabular}

animals $(P=0.0170)$ (Table 2). Mean blood glucose was 126.2 $\pm 5.191(n=35)$ for hypothermic and 108.9 $\pm 4.044(n=25)$ for normothermic animals.

\section{Sensorimotor Outcomes}

No significant differences were observed between the experimental groups (normothermia group without Epo, normothermia group with Epo, hypothermia group without Epo, and hypothermia group with Epo) for behavioral outcomes at either 2 or 6 wk after HI using the cylinder-rearing test. Unimpaired paw preference means were similar among all groups (Figure 1), with no differences between groups in terms of calculated overall
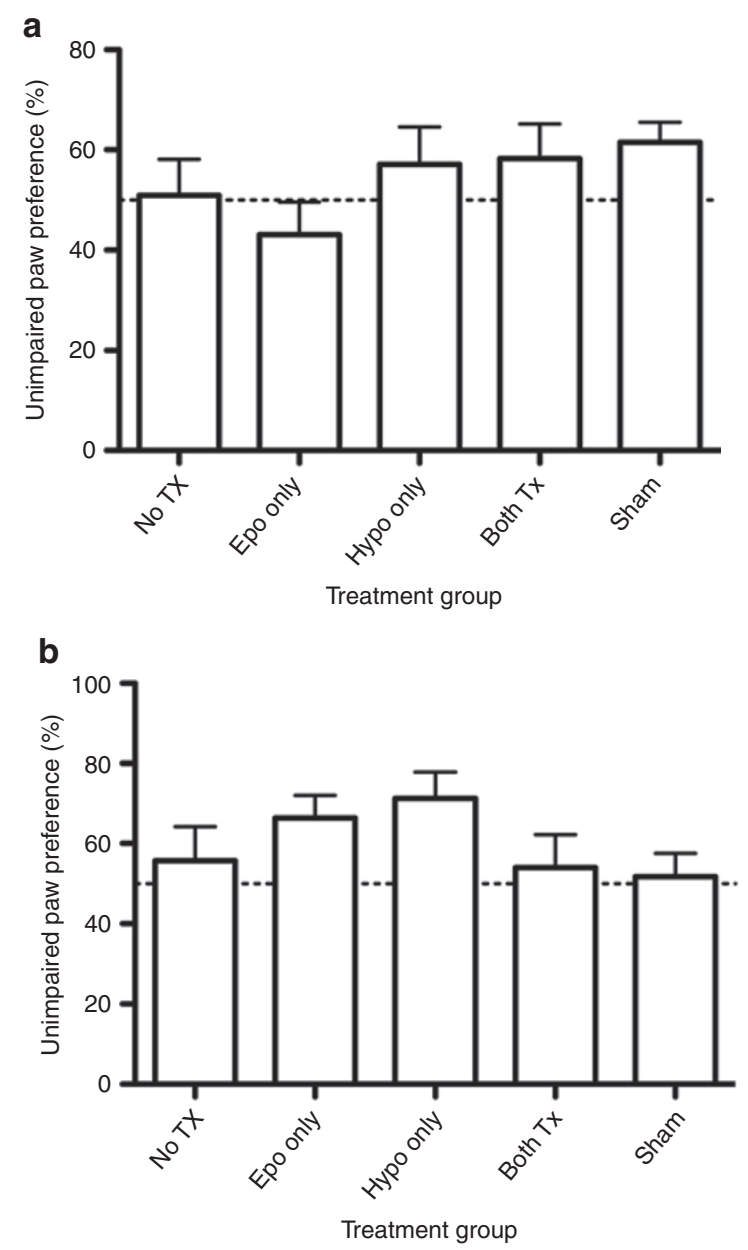

Figure 1. Behavioral testing at (a) $2 \mathrm{wk}$ and (b) $6 \mathrm{wk}$ after $\mathrm{HI}$ or sham surgery at P7. Percentage of unimpaired forepaw preference for full rears in each treatment group was calculated as: number of (right contacts + $1 / 2$ bilateral contacts)/number of (right + left + bilateral contacts) $\times 100$. Mean ( \pm SEM) values with error bars are shown for each group. No significant differences between experimental groups and sham-treated animals were detected, with similar unimpaired paw preference means (one-way ANOVA with Bonferroni post hoc testing for multiple comparisons; $n=10$ for no Tx, $n=10$ for Epo only, $n=12$ for Hypo only, $n=13$ for both Tx, $n=5$ for sham surgery at $2 \mathrm{wk} ; n=8$ for no Tx, $n=12$ for Epo only, $n=9$ for Hypo only, $n=10$ for both Tx, $n=14$ for sham surgery at $6 \mathrm{wk}$ ). No $\mathrm{Tx}=\mathrm{HI}$ with no treatment given; Epo only $=\mathrm{HI}$ with Epo treatment; Hypo only $=\mathrm{HI}$ with hypothermia treatment; Both $\mathrm{Tx}=\mathrm{HI}$ with combination treatment; Sham = sham surgery. Epo, erythropoietin; $\mathrm{HI}$, hypoxic-ischemic; Hypo, hypothermia. 
activity level (data not shown). Multifactorial analysis using linear regression demonstrated no differences in paw preference based on sex.

\section{Histopathological Outcomes}

No significant differences were detected between the four experimental groups for brain damage score outcomes at either 2 or 6 wk after HI (Figure 2). Moreover, histology and behavioral performance were not correlated (data not shown). However,
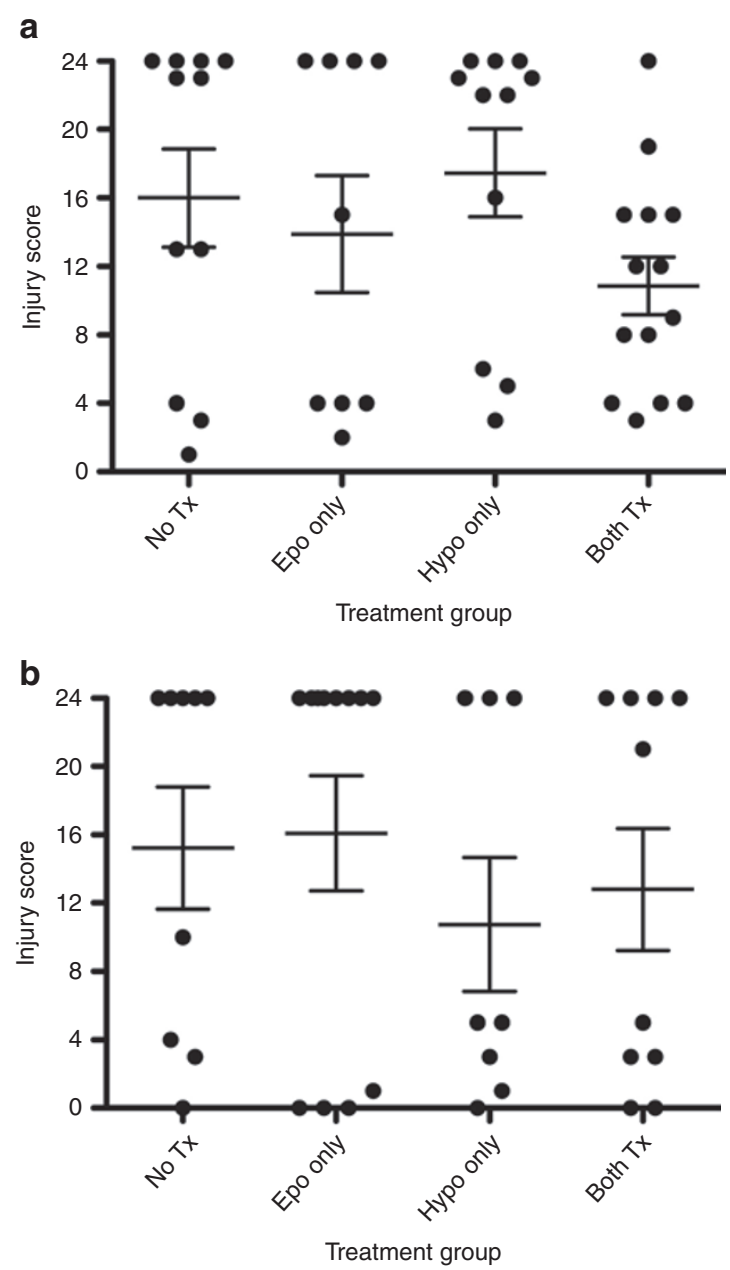

Figure 2. Histopathological scoring of cresyl violet and Perl's iron-stained sections throughout the brain at (a) $2 \mathrm{wk}$ and (b) $6 \mathrm{wk}$ following $\mathrm{HI}$ or sham surgery. The cingulate cortex, parietal cortex, caudate/putamen, hippocampus, and thalamus were scored as follows: $0=$ no injury, $1=$ minimal cell loss manifested by scattered shrunken neurons and glia, $2=$ moderate cell loss with infarction in a columnar distribution in the cortex with concomitant gliosis or shrunken hippocampus with cell loss throughout Sommer's sector, and 3 = severe cell loss and gliosis with cystic infarction. Data are presented as mean ( \pm SEM) values. There was no significant difference between injured animals regardless of treatment group (Kruskal-Wallis test with Dunn's multiple comparison), although there was a statistically significant sex-specific difference in injury score for animals treated with Epo $(P=0.041)$ (multivariate analysis with linear regression to test for a sex-by-treatment interaction using nonparametric bootstrap). No Tx = HI with no treatment given; Epo only = HI with Epo treatment; Hypo only = HI with hypothermia treatment; Both $\mathrm{Tx}=\mathrm{HI}$ with combination treatment. Epo, erythropoietin; HI, hypoxic-ischemic; Hypo, hypothermia. multifactorial analysis using linear regression showed a sexbased difference in histopathological outcome in male animals treated with Epo only $(P=0.041)$.

\section{DISCUSSION}

In this study, we assessed the efficacy of combined hypothermia and Epo treatment on short-term functional and histological outcomes in a rat model of neonatal $\mathrm{HI}$ at postnatal day 7 (P7). This was a negative study, with no benefit observed with hypothermia or combination therapy on either neuropathology score or sensorimotor outcome. There was also no correlation between histology and function in these animals. A sex-specific effect of treatment with Epo on histological outcome alone was detected on linear regression analysis, showing benefit of Epo in male animals after HI, but there were no sex-specific differences in the other experimental groups or on sensorimotor outcome.

Epo has previously been shown to improve histology and function after neonatal rodent stroke when using a similar treatment protocol (13). However, this regimen was not beneficial in this study's model of neonatal HI and may have used a suboptimal dosage or number of doses. The literature regarding the usage of Epo in neonatal rodent HI is quite heterogeneous; different timing and dosage of Epo have had variable results. For example, a single dose of Epo at $1,000 \mathrm{U} / \mathrm{kg}$ was shown to improve short- and long-term brain injury and behavioral outcomes (14). Other studies reported benefits of Epo when administered in multiple doses of 500-1,000 U/kg, with no further benefit observed at 2,000 U/kg (16). Another study comparing high-dose Epo protocols reported benefit with doses ranging from 5,000 to $30,000 \mathrm{U} / \mathrm{kg}$, with most benefit arising from multiple doses of 5,000 U/kg (23). Of note, some studies demonstrate improvement in sensorimotor outcomes without significantly improving histopathological outcomes. For example, a recent study showed that delayed and repeated dosing of $1,000 \mathrm{U} / \mathrm{kg}$ improved sensorimotor performance and enhanced oligodendrogliosis, but was ineffective at decreasing infarct volume (26). Similarly, three daily doses of 2,500 U/kg improved functional outcomes into adulthood without improving histopathology (27). Recently, enhanced benefit was noted with a nano-derivative of erythropoietin in a neonatal rat stroke model, which may facilitate blood-brain barrier crossing (28). Indeed, the optimal regimen of treatment with Epo in this model remains to be elucidated, as the ideal dosage, number of doses, and timing of administration are still not clear.

In addition, no observable benefit from hypothermia was noted in this study. Of note, although rats at age P7-P10 are often used to model the term newborn brain, P7 may actually represent a more immature (late-preterm) brain, and P10 may more accurately represent the full-term brain (29). In fact, earlier studies have shown equivocal benefit with target temperatures ranging from 31 to $34^{\circ} \mathrm{C}$ for a total of $3 \mathrm{~h}$ (8), with only Thoresen et al. (30) showing improvement in histopathological outcomes when cooling to $32^{\circ} \mathrm{C}$ rectal temperature for $3 \mathrm{~h}$. Bona et al. (24) reported that cooling for $6 \mathrm{~h}$ following $\mathrm{HI}$ resulted in improved brain injury scores and sensorimotor outcomes in female 


\section{Hypothermia and Epo in neonatal rat HI}

animals. More recent studies have focused on longer duration of hypothermia, with mixed results. Hypothermia for $10 \mathrm{~h}$ to $32^{\circ} \mathrm{C}$ and $26^{\circ} \mathrm{C}$ did not improve striatal damage (22), whereas others demonstrated short-term histological improvements with the same duration of hypothermia to $30^{\circ} \mathrm{C}(31)$. Even longer duration of hypothermia has been evaluated, with histological and functional improvement following 24-48 h of hypothermia to $30^{\circ} \mathrm{C}(20)$ except in severely injured animals (32). Although $72 \mathrm{~h}$ of cooling to $33^{\circ} \mathrm{C}$ has become standard of care for human neonates with suspected HIE, the optimal protocol for hypothermia in the neonatal rat model of HI has, like Epo dosing, yet to be completely identified. It is possible that more benefit can be gained from deeper or more prolonged hypothermia.

Other methodological issues have been raised regarding the administration of hypothermia in rodents. Most studies induce hypothermia via temperature-controlled ambient air temperatures or by titration of ambient air temperature to reach a target rectal temperature. In our model, hypothermia was administered with air cylinders partially submerged in a water bath adjusted to $32^{\circ} \mathrm{C}$, whereas normothermic animals were kept in air cylinders submerged in a $37^{\circ} \mathrm{C}$ water bath. Mean cranial skin temperature of hypothermic animals was $\sim 31^{\circ} \mathrm{C}$, whereas that of normothermic animals kept was $34^{\circ} \mathrm{C}$, raising the issue of whether normothermic animals were actually kept in mildly hypothermic conditions. A preferable strategy may have been titration of water bath temperature to maintain ambient air temperature within cylinders.

An additional concern regarding hypothermic temperature regulation involves the stress response to cooling. In this experiment, we found a significant difference in blood glucose following temperature treatment not noted in previous studies (24), raising the issue of potential compensatory responses to cooling that might interfere with neuroprotection, such as agitation, tachycardia, and shivering. Many studies demonstrated hypothermic neuroprotective effects in humans and piglets administered cooling under intensive-care settings, including anesthesia. A notable study in piglets demonstrated that lack of anesthesia during cooling resulted in higher stress levels and abolished the neuroprotective benefit of hypothermia (33). Indeed, there may even be detrimental effects from unanesthetized hypothermia that we were unable to detect in this study. In addition, the use of morphine during hypothermia in humans has been shown to be neuroprotective synergistically (34). In light of these studies, potential improvements to the current experimental protocol may include sedative and analgesic administration.

In contrast with some previous studies, no correlation between histological injury and sensorimotor performance was observed $(11,13)$. Treated animals did not differ from untreated animals or sham-treated animals in forepaw preference in the cylinder-rearing test, making interpretation of treatments difficult. It is unclear why there was a lack of correlation between histology and function, although the heterogeneity of injury patterns seen here in HI animals, with mild and severely injured subjects mixed with relatively few moderately injured animals, may cloud the results. Although such a wide range of injury has been historically reported with this model
(35), the high incidence of severe damage may represent the inaccuracy of this rodent model in determining short-term sensorimotor outcomes. There is probably a threshold for damage beyond which no treatment is beneficial $(27,32)$, as well as a target group with moderate damage that will likely benefit the most from treatment. The effects of therapy may be more pronounced if animals with moderate injury can be identified and selected for treatment, as performed in other studies using magnetic resonance imaging (20). In addition, it is also possible that the cylinder-rearing test was insufficiently sensitive to detect differences between groups, or that all HI-injured animals regardless of injury severity were able to functionally compensate in this assay. Other tests of sensorimotor and cognitive function, and testing at later time points, may better demonstrate differences between treated groups. Finally, population-level differences in paw preference may also have obscured results, because sham-treated animals in this study appeared to have a right-paw preference that has not been previously reported in other studies.

Analysis of sex-based differences in outcome was performed in response to many studies in the literature that report sex-specific neuroprotective effects for a number of therapies, including hypothermia (24) and Epo $(25,36)$. In this study, we found improvement in histopathological outcomes after the treatment with Epo that was specific to male animals. Brain development is known to be dimorphic and influenced by circulating hormones and differential gene expression even in the absence of hormonal influences (37), and this likely underlies sex differences in brain injury and response to therapies, although the specifics of these effects remain largely unknown (38). Neither sex-specific effects on sensorimotor outcomes in the different experimental groups after multifactorial analysis nor sex-specific differences on histopathological outcome in animals that received hypothermia alone or combination therapy were observed.

In this study, although we were unable to detect any significant benefit from hypothermia, Epo, or combination therapy, no adverse effects were associated with combined treatment. Future studies may require slightly older animals (P10) to more closely model the full-term newborn brain and to show the possible benefit of combination therapy. To control for injury severity and its effects on treatment response, magnetic resonance imaging following injury may help to stratify animals into mild, moderate, and severe injury groups. In addition, determination of the long-term functional response with a wider range of sensorimotor and cognitive testing will be necessary to evaluate the true benefits of combination therapy.

\section{METHODS}

\section{Animal Model of $\mathrm{HI}$}

Animal experiments were performed in accordance with the guidelines from the National Institutes of Health for humane handling of animals and were approved by the University of California, San Francisco Institutional Animal Care and Use Committee. Surgical procedures were performed in P7 Sprague-Dawley rats $(n=85)$. Rats were subjected to the Vannucci model (35) for HI. Briefly, under isofluorane anesthesia (Butler Schein Animal Health Supply, Reno, NV) administered at $3 \%$ for induction and $1.5 \%$ for maintenance, the right common carotid artery was permanently ligated following midline 
incision. Following a 90-min recovery period, rats were exposed to hypoxia for $120 \mathrm{~min}$ ( $8 \%$ oxygen and balance nitrogen) while being maintained at $37^{\circ} \mathrm{C}$. Sham-treated animals $(n=19)$ were anesthetized and had the common carotid artery exposed without ligation.

\section{Postischemic Temperature Modulation and Epo Administration}

Rats were divided into the following groups: sham-treated group, normothermia group without Epo, normothermia group with Epo, hypothermia group without Epo, or hypothermia group with Epo. Sham-treated animals were returned to the dam with no further intervention. Immediately following hypoxia, either vehicle $(0.1 \%$ bovine serum albumin (Sigma-Aldrich, St Louis, MO) in saline) or recombinant human Epo (R\&D Systems, Minneapolis, MN) was injected intraperitoneally. In the Epo-treated rats, an initial dose of 1,000 U/kg was given. Rats were then allowed to recover with the dam for $1 \mathrm{~h}$, after which, temperature modulation was initiated. Repeat doses of either vehicle or Epo were administered at $24 \mathrm{~h}$ and $7 \mathrm{~d}$ after injury.

For temperature modulation, normothermic pups were kept in separate air cylinders maintained in a water bath at $37^{\circ} \mathrm{C}$, whereas hypothermic pups were kept in individual air cylinders maintained in a water bath at $32^{\circ} \mathrm{C}$, for a total of $8 \mathrm{~h}$. After $4 \mathrm{~h}$, pups were gavage fed with $0.5 \mathrm{ml}$ of milk formula. Cranial skin temperature was measured at 4 and $8 \mathrm{~h}$ during temperature modulation using an infrared thermometer (Extech, Waltham, MA).

\section{Additional Monitoring}

Blood glucose was measured before $\mathrm{HI}$ and following temperature modulation. Weights of the animals were monitored for $2 \mathrm{wk}$ following $\mathrm{HI}$ and at killing, either 2 or $6 \mathrm{wk}$ following $\mathrm{HI}$.

\section{Sensorimotor Testing}

Rats were assessed at 2 and $6 \mathrm{wk}$ after HI using the cylinder-rearing test. Asymmetry in the use of the forelimbs was determined by analyzing the movements of each rat during exploratory activity within a transparent cylinder of a diameter of $20 \mathrm{~cm}$. Two mirrors were positioned behind the cylinder, and each animal was video-recorded for at least $20 \mathrm{~min}$. Initial forepaw placement of each weight-bearing full rear was recorded. "Bilateral" was recorded in cases of simultaneous contact with both forepaws. Data are presented as the scores of limb-use asymmetry according to the following formula: [number of (right contacts $+1 / 2$ bilateral contacts)/number of (right + left + bilateral contacts) $] \times$ 100. This resulted in an asymmetry score that reflected preference of the unimpaired (right) limb. This score was higher for animals with poorer performance, i.e., increased preference of the unimpaired (right) limb and decreased use of the affected (left) limb. The timing and frequency of full rears were also recorded. Overall activity level was calculated by measuring time to initiate first full rear, time to complete four full rears, and number of full rears performed in the initial $10 \mathrm{~min}$.

\section{Histology}

For histopathological examination, following behavioral testing, animals were anesthetized with Euthasol (Butler Schein Animal Health Supply) and killed at 2 or $6 \mathrm{wk}$ following HI by transcardiac perfusion with ice-cold $4 \%$ paraformaldehyde (Sigma-Aldrich) in $0.1 \mathrm{~mol} / \mathrm{l}$ phosphate-buffered saline. Brains were carefully removed and postfixed overnight, equilibrated in $30 \%$ sucrose in $0.1 \mathrm{~mol} / \mathrm{l}$ phosphatebuffered saline and left at $4{ }^{\circ} \mathrm{C}$ in $0.1 \mathrm{~mol} / \mathrm{l}$ phosphate buffer. Using a vibratome (Leica Microsystems, Wetzlar, Germany), 50- $\mu \mathrm{m}$ coronal sections were made. Every third section was mounted and air-dried, stained with cresyl violet and dehydrated in graded ethanol solutions, cleared in Citrisolv (Fisher Scientific, Hampton, NH), and coverslipped in Permount (Fisher Scientific). Another subset of sections were stained with Perl's iron stain, then mounted, air-dried, dehydrated, and coverslipped as above.

\section{Histopathological Measurements}

Histopathological scoring of cresyl violet and Perl's iron-stained sections was done blindly. Sections were assessed rostrally from the genu of the corpus callosum through the hippocampus caudally. Regions studied included the cingulate and parietal cortices, caudate/putamen, hippocampus, and thalamus. Scoring was performed as follows: $0=$ no injury, $1=$ minimal cell loss manifested by scattered shrunken neurons and glia, 2 = moderate cell loss with infarction in a columnar distribution in the cortex with concomitant gliosis or shrunken hippocampus with cell loss throughout Sommer's sector, and $3=$ severe cell loss and gliosis with cystic infarction (39).

\section{Statistical Analysis}

The Kruskal-Wallis test with Dunn's multiple comparison was used for the histological scoring of brain injury, and one-way ANOVA with Bonferroni testing was used for the functional testing by cylinder rearing. We fit a regression model to the damage scores and limb preference and formally tested for a sex-by-treatment interaction to examine more rigorously whether treatment effects varied by sex. We also performed a linear regression analysis using the nonparametric bootstrap to form tests and confidence intervals to address the potentially nonnormal distribution of the data. $P<0.05$ was considered to be significant. Analysis was performed with Prism 4.0 (GraphPad Software, La Jolla, CA) and Stata version 11 (Stata, College Station, TX).

\section{STATEMENT OF FINANCIAL SUPPORT}

This study was supported by National Institutes of Health (NIH) R01 NS 33997 and NS 35902 grants to D.M.F., NIH 1 K08NS064094 grant to F.F.G., and the Howard Hughes Medical Institute Medical Research Fellows Program to A.Y.F.

\section{ACKNOWLEDGMENTS}

The authors thank David Glidden for statistical assistance.

\section{REFERENCES}

1. Jacobs S, Hunt R, Tarnow-Mordi W, Inder T, Davis P. Cooling for newborns with hypoxic ischaemic encephalopathy. Cochrane Database Syst Rev 2007:CD003311.

2. Gonzalez FF, Ferriero DM. Therapeutics for neonatal brain injury. Pharmacol Ther 2008;120:43-53.

3. Gluckman PD, Wyatt JS, Azzopardi D, et al. Selective head cooling with mild systemic hypothermia after neonatal encephalopathy: multicentre randomised trial. Lancet 2005;365:663-70.

4. Shankaran S, Laptook AR, Ehrenkranz RA, et al. Whole-body hypothermia for neonates with hypoxic-ischemic encephalopathy. N Engl J Med 2005;353:1574-84.

5. Azzopardi DV, Strohm B, Edwards AD, et al. Moderate hypothermia to treat perinatal asphyxial encephalopathy. N Engl J Med 2009;361:1349-58.

6. Drury PP, Bennet L, Gunn AJ. Mechanisms of hypothermic neuroprotection. Semin Fetal Neonatal Med 2010;15:287-92.

7. Thoresen M, Penrice J, Lorek A, et al. Mild hypothermia after severe transient hypoxia-ischemia ameliorates delayed cerebral energy failure in the newborn piglet. Pediatr Res 1995;37:667-70.

8. Laptook AR, Corbett RJ, Sterett R, Burns DK, Tollefsbol G, Garcia D. Modest hypothermia provides partial neuroprotection for ischemic neonatal brain. Pediatr Res 1994;35(4 Pt 1):436-42.

9. Gunn AJ, Gunn TR, de Haan HH, Williams CE, Gluckman PD. Dramatic neuronal rescue with prolonged selective head cooling after ischemia in fetal lambs. J Clin Invest 1997;99:248-56.

10. Perlman JM, Wyllie J, Kattwinkel J, et al.; Neonatal Resuscitation Chapter Collaborators. Part 11: Neonatal resuscitation: 2010 International Consensus on Cardiopulmonary Resuscitation and Emergency Cardiovascular Care Science With Treatment Recommendations. Circulation 2010;122:16 Suppl 2:S516-38.

11. Chang YS, Mu D, Wendland M, et al. Erythropoietin improves functional and histological outcome in neonatal stroke. Pediatr Res 2005;58: 106-11.

12. Gonzalez FF, McQuillen P, Mu D, et al. Erythropoietin enhances longterm neuroprotection and neurogenesis in neonatal stroke. Dev Neurosci 2007;29:321-30.

13. Gonzalez FF, Abel R, Almli CR, Mu D, Wendland M, Ferriero DM. Erythropoietin sustains cognitive function and brain volume after neonatal stroke. Dev Neurosci 2009;31:403-11. 
14. Kumral A, Uysal N, Tugyan K, et al. Erythropoietin improves long-term spatial memory deficits and brain injury following neonatal hypoxia-ischemia in rats. Behav Brain Res 2004;153:77-86.

15. Sun Y, Calvert JW, Zhang JH. Neonatal hypoxia/ischemia is associated with decreased inflammatory mediators after erythropoietin administration. Stroke 2005;36:1672-8.

16. Iwai M, Cao G, Yin W, Stetler RA, Liu J, Chen J. Erythropoietin promotes neuronal replacement through revascularization and neurogenesis after neonatal hypoxia/ischemia in rats. Stroke 2007;38:2795-803.

17. Xiong T, Qu Y, Mu D, Ferriero D. Erythropoietin for neonatal brain injury: opportunity and challenge. Int J Dev Neurosci 2011;29:583-91.

18. Elmahdy H, El-Mashad AR, El-Bahrawy H, El-Gohary T, El-Barbary A, Aly $\mathrm{H}$. Human recombinant erythropoietin in asphyxia neonatorum: pilot trial. Pediatrics 2010;125:e1135-42.

19. Zhu C, Kang W, Xu F, et al. Erythropoietin improved neurologic outcomes in newborns with hypoxic-ischemic encephalopathy. Pediatrics 2009;124:e218-26.

20. Lee BS, Woo CW, Kim ST, Kim KS. Long-term neuroprotective effect of postischemic hypothermia in a neonatal rat model of severe hypoxic ischemic encephalopathy: a comparative study on the duration and depth of hypothermia. Pediatr Res 2010;68:303-8.

21. Trescher WH, Ishiwa S, Johnston MV. Brief post-hypoxic-ischemic hypothermia markedly delays neonatal brain injury. Brain Dev 1997;19:326-38.

22. Covey MV, Oorschot DE. Effect of hypothermic post-treatment on hypoxic-ischemic striatal injury, and normal striatal development, in neonatal rats: a stereological study. Pediatr Res 2007;62:646-51.

23. Kellert, McPherson RJ, Juul SE. A comparison of high-dose recombinant Epo treatment regimens in brain-injured neonatal rats. Pediatr Res 2007;61:451-5.

24. Bona E, Hagberg H, Løberg EM, Bågenholm R, Thoresen M. Protective effects of moderate hypothermia after neonatal hypoxia-ischemia: shortand long-term outcome. Pediatr Res 1998;43:738-45.

25. Fan X, Heijnen CJ, van der KOOIJ MA, Groenendaal F, van Bel F. Beneficial effect of erythropoietin on sensorimotor function and white matter after hypoxia-ischemia in neonatal mice. Pediatr Res 2011;69:56-61.

26. Iwai M, Stetler RA, Xing J, et al. Enhanced oligodendrogenesis and recovery of neurological function by erythropoietin after neonatal hypoxic/ ischemic brain injury. Stroke 2010;41:1032-7.
27. Demers EJ, McPherson RJ, Juul SE. Erythropoietin protects dopaminergic neurons and improves neurobehavioral outcomes in juvenile rats after neonatal hypoxia-ischemia. Pediatr Res 2005;58:297-301.

28. Chen H, Spagnoli F, Burris M, et al. Nanoerythropoietin is 10-times more effective than regular erythropoietin in neuroprotection in a neonatal rat model of hypoxia and ischemia. Stroke 2012;43:884-7.

29. Hagberg H, Ichord R, Palmer C, Yager JY, Vannucci SJ. Animal models of developmental brain injury: relevance to human disease. A summary of the panel discussion from the Third Hershey Conference on Developmental Cerebral Blood Flow and Metabolism. Dev Neurosci 2002;24:364-6.

30. Thoresen M, Bågenholm R, Løberg EM, Apricena F, Kjellmer I. Posthypoxic cooling of neonatal rats provides protection against brain injury. Arch Dis Child Fetal Neonatal Ed 1996;74:F3-9.

31. Zhu C, Wang X, Cheng X, et al. Post-ischemic hypothermia-induced tissue protection and diminished apoptosis after neonatal cerebral hypoxiaischemia. Brain Res 2004;996:67-75.

32. Nedelcu J, Klein MA, Aguzzi A, Martin E. Resuscitative hypothermia protects the neonatal rat brain from hypoxic-ischemic injury. Brain Pathol 2000;10:61-71.

33. Thoresen M, Satas S, Løberg EM, et al. Twenty-four hours of mild hypothermia in unsedated newborn pigs starting after a severe global hypoxicischemic insult is not neuroprotective. Pediatr Res 2001;50:405-11.

34. Simbruner G, Mittal RA, Rohlmann F, Muche R. Systemic hypothermia after neonatal encephalopathy: outcomes of neo.nEURO.network RCT. Pediatrics 2010;126:e771-8.

35. Rice JE 3rd, Vannucci RC, Brierley JB. The influence of immaturity on hypoxic-ischemic brain damage in the rat. Ann Neurol 1981;9:131-41.

36. Wen TC, Rogido M, Peng H, Genetta T, Moore J, Sola A. Gender differences in long-term beneficial effects of erythropoietin given after neonatal stroke in postnatal day-7 rats. Neuroscience 2006;139:803-11.

37. Hurn PD, Vannucci SJ, Hagberg H. Adult or perinatal brain injury: does sex matter? Stroke 2005;36:193-5.

38. Gonzalez FF, Fang A, Ferriero DM. Is erythropoietin the answer? Pediatr Res 2011;69:2-3.

39. Ferriero DM, Sheldon RA, Black SM, Chuai J. Selective destruction of nitric oxide synthase neurons with quisqualate reduces damage after hypoxiaischemia in the neonatal rat. Pediatr Res 1995;38:912-8. 